\title{
Analysis of transcription factor Stk40 expression and function during mouse pre-implantation embryonic development
}

\author{
JUNQIANG ZHANG $^{1,2^{*}}$, JUANJUAN ZHANG $^{2 *}$, CHUN ZHAO $^{2}$, RONG SHEN $^{2}$, \\ XIRONG GUO ${ }^{2}$, CHAOJUN $\mathrm{LI}^{3}$, XIUFENG LING ${ }^{2 *}$ and CHANG LIU ${ }^{1}$ \\ ${ }^{1}$ Jiangsu Key Laboratory for Molecular and Medical Biotechnology, College of Life Sciences, Nanjing Normal University, \\ Nanjing, Jiangsu 210046; ${ }^{2}$ Department of Reproduction, Nanjing Maternity and Child Health Care Hospital, \\ Affiliated to Nanjing Medical University, Nanjing Medical University, Nanjing, Jiangsu 210004; \\ ${ }^{3}$ Key Laboratory of Model Animals for Disease Study of Ministry of Education, Model Animal Research Centre, \\ Nanjing University, Nanjing, Jiangsu 210093, P.R. China
}

Received April 9, 2013; Accepted November 14, 2013

DOI: $10.3892 / \mathrm{mmr} .2013 .1828$

\begin{abstract}
Determining the molecular mechanisms in the regulation of early embryonic development is crucial for assisted reproductive technology clinical applications. Serine/threonine protein kinase 40 (Stk40) is a member of the serine/threonine kinase family. It is essential in diverse signaling pathways associated with a wide range of cellular activities, including proliferation, differentiation, survival and apoptosis. However, its involvement and molecular mechanisms in pre-implantation embryonic development have not been well-defined. In the present study, it was demonstrated that Stk40 was involved in the development of mouse pre-implantation embryos. Immunofluorescence and confocal microscopy analyses showed that Stk40 was equally expressed in the nuclei and cytoplasm during all stages of pre-implantation mouse embryos of imprinting control region mice. Reverse transcription-polymerase chain reaction showed a significantly higher transcription rate of Stk40 mRNA in the two-cell stage. The results demonstrated that Stk40 downregulation by microinjection of small interfering RNA into the mouse zygote markedly decreased the blastulation compared with that in the control (Stk40i-1 vs. control: $65.2 \%$ and $77.0 \%$,
\end{abstract}

Correspondence to: Professor Xiufeng Ling, Department of Reproduction, Nanjing Maternity and Child Health Care Hospital, Affiliated to Nanjing Medical University, 123 Tianfei Lane, MoChou Road, Nanjing, Jiangsu 210004, P.R. China

E-mail: xiufenglingBM@163.com

Professor Chang Liu, Jiangsu Key Laboratory for Molecular and Medical Biotechnology, College of Life Sciences, Nanjing Normal University, 1 WenYuan Road, Nanjing, Jiangsu 210046, P.R. China E-mail: changliuBM@163.com

"Contributed equally

Key words: Stk40, pre-implantation, gene knockdown, blastulation
$\mathrm{P}<0.05$ and Stk40i-2 vs. control: $49.8 \%$ and $70.1 \%$, respectively, $\mathrm{P}<0.05)$. In addition, silencing of Stk40 significantly increased the transcription rate of reticulocalbin-2, whereas that of the homeobox protein, $\mathrm{Cdx} 2$, was decreased. In conclusion, the results suggested that Stk40 may be critical in the development of pre-implantation embryos.

\section{Introduction}

The development of mouse pre-implantation embryos involves substantial cell proliferation and differentiation, and cell lineages are established early during embryonic development. Various studies, over the past few years, have identified key transcription factor networks during mouse pre-implantation embryonic development (1-5). These transcription factors, including octamer-binding transcription factor 4 (Oct4), Sox2, Kruppel-like factor 4 (Klf4) and c-Myc have been demonstrated to reprogram somatic cells in vitro leading to the generation of pluripotent stem cells derived from mouse embryonic fibroblasts (6). Therefore, transcription factors are essential regulators of the transition from germ-cell-type to embryo-type gene expression and are key in determining cell characteristics (7). In addition, numerous studies have shown that epigenetic regulation is a decisive factor in pre-implantation embryo development $(8,9)$ and each embryonic developmental stage is characterized by a specific epigenetic pattern (10). It has been determined that in vitro developmental block occurs in different species at different stages of embryonic development, which is concurrent with embryonic genome activation (11). The developmental block in mouse, bovine and human embryos occurs at the two-cell, four-cell and four- to eight-cell stages (12).

In clinically assisted reproduction practices, human embryos cultured in vitro are sensitive to developmental arrest and the completed blastocyst formation rate is only 50\% (13); however, the reasons for this are not well understood. Although improvements in in vitro culture conditions have greatly enhanced the embryo integrity, the in vitro fertilization (IVF) success rates are not ideal. Thus, increased knowledge of the molecular mechanisms regulating oogenesis, fertilization, 
cleavage, blastocyst formation and implantation is crucial for future assisted reproductive technology improvements. The Serine/threonine protein kinase 40 (Stk40) Stk40 gene has been identified previously, by a low density array analysis, to be one of the development-related transcription factors in mouse embryonic pre-implantation development. This gene was initially defined as a SINK-homologous serine/threonine kinase, designated as SHIK, which negatively regulates NF- $\mathrm{B}$ and p53-mediated transcription (14). In a previous ChIP-chip assay, the promoter of Stk40 was demonstrated to associate with Oct4 as well as Sox2 and Nanog (15). In addition, it was shown that Stk40, a target gene of Oct4 transcription, was able to activate the extracellular signal-regulated kinase (ERK)/mitogen-activated protein kinase (MAPK) signaling pathway and induce embryonic stem cell differentiation into extraembryonic-endoderm (ExEn) cell lineages through reticulocalbin-2 (Rcn2) (16). However, its function and mechanism of gene regulation in early embryonic development remains to be further elucidated.

In the present study, the expression and localization of the Stk40 gene was investigated during mouse embryonic development. Downregulation of Stk40 indicated that this gene was essential in mouse embryogenesis, particularly in blastocyst formation. In addition, the results suggested an association between Stk40 and the ERK/MAPK signaling pathway.

\section{Materials and methods}

Chemicals. 4-(2-hydroxyethyl)-1-piperazineethanesulfonic acid (HEPES)-buffered Chatot, Ziomet and Bavister (CZB) medium was purchased from Sigma-Aldrich (St. Louis, MO, USA), polyclonal Stk40 antibodies were purchased from Jingtiancheng Biotech Co., Ltd. (Beijing, China).

Embryo collection and culture. A total of 200 Imprinting control region (ICR) female mice (Shanghai Slac Labortory Animal Co., Ltd., Shanghai, China) were maintained in a controlled environment of $20-22^{\circ} \mathrm{C}$ and $12 / 12$-h light/dark cycle, with $50-70 \%$ humidity and access to food and water ad libitum. Animal care and experimental procedures were conducted in accordance with the Animal Research Committee guidelines of the Nanjing Medical University (Nanjing, China). For the collection of zygotes, female ICR mice (age, 8 weeks) were injected with human chorionic gonadotropin and mated with ICR male mice shortly following the injection. After $15-17 \mathrm{~h}$, zygotes were collected from the oviducts of the mice and transferred onto a HEPES-buffered CZB medium. Cumulus cells were dispersed by treatment with $1 \mathrm{mg} / \mathrm{ml}$ hyaluronidase in HEPES-CZB. Cumulus-free zygotes were washed with HEPES-CZB medium and cultured in CZB medium until they grew to the later early embryo stages at $37^{\circ} \mathrm{C}$ in a $5 \% \mathrm{CO}_{2}$ atmosphere. The study was approved by the Ethical committee of Nanjing Normal University, Nanjing Maternity and Child Health Care Hospital Affiliated to Nanjing Medical University and of the Medical School of Nanjing University, Nanjing University (Nanjing, China).

Immunoblotting analysis. Immunoblotting was performed to analyze the valence of made-to-order Stk40 antibodies (Jingtiancheng Biotech Co., Ltd.). The ovary proteins of adult
ICR mice were separated by sodium dodecyl sulfate-polyacrylamide gel electrophoresis and then electrophoresed onto polyvinylidene fluoride membranes. Following the transfer, the membranes were blocked in Tris-buffered saline with $0.1 \%$ Tween-20 (TBST) containing 5\% skimmed milk for $2 \mathrm{~h}$, followed by overnight incubation at $4{ }^{\circ} \mathrm{C}$ with rabbit polyclonal anti-Stk40 antibodies (dilution, 1:500; NFB001; Jingtiancheng Biotech Co., Ltd.). Following three $10 \mathrm{~min}$ washes with TBST, the membranes were incubated for $1 \mathrm{~h}$ at $37^{\circ} \mathrm{C}$ with horseradish peroxidase-conjugated goat anti-rabbit $\operatorname{IgG}$ (dilution, 1:1000; Zhongshan Biotech, Beijing, China). The membranes were then processed using an enhanced chemiluminescence detection system (Model 920; Amersham Biosciences, Piscataway, NJ, USA).

Immunofluorescence and confocal microscopy. For staining of Stk40, the embryos were fixed in $4 \%$ paraformaldehyde in phosphate-buffered saline (PBS; $\mathrm{pH} 7.4$ ) for $\geq 30 \mathrm{~min}$ at room temperature. Subsequent to permeabilization with $0.5 \%$ Triton X-100 at room temperature for $20 \mathrm{~min}$, the embryos were incubated with $1 \%$ bovine serum albumin-supplemented PBS for $1 \mathrm{~h}$ and incubated overnight at $4^{\circ} \mathrm{C}$ with rabbit anti-Stk40 antibodies (dilution, 1:1000; Jingtiancheng Biotech Co., Ltd.). Following three 5 min washes with PBS containing $0.1 \%$ Tween-20 and $0.01 \%$ Triton X-100, the embryos were labeled with fluorescein isothiocyanate-conjugated goat anti-rabbit IgG (dilution, 1:100; Zhongshan Biotech) for $1 \mathrm{~h}$ at room temperature. Subsequent to another three washes in PBS containing $0.1 \%$ Tween-20 and $0.01 \%$ Triton X-100, the embryos were stained with propidium iodide $(10 \mu \mathrm{g} / \mathrm{ml}$ in PBS). The embryos were mounted on glass slides and examined under a confocal laser scanning microscope (Zeiss LSM 510 META, Zeiss, Jena, Germany).

Stk40 short interfering RNA (siRNA) microinjection and in vitro culture. Stk40-siRNA (MSS232404, MSS232405 and MSS292686) was purchased from Invitrogen Life Technologies (Carlsdbad, CA, USA). MISSION ${ }^{\circledR}$ siRNA is a heterogeneous mixture of siRNAs that target the same mRNA sequence. This multiple silencing approach leads to highly specific and effective gene silencing. The Stk40-siRNA was stored at $-20^{\circ} \mathrm{C}$ at a concentration of $200 \mathrm{ng} / \mu \mathrm{l}$. Approximately $10 \mathrm{pl}$ of individual siRNA was microinjected into the cytoplasm of the zygotes, which were cultured in CZB medium for further observation. The developmental status of each group was identified and analyzed using a Nikon Eclipse TE2000-S microscope.

Reverse-transcription polymerase chain reaction (real-time PCR). The primer sequences of Stk40 and related transcription factors are shown in Table I. siRNA-injected and control oocytes were paired to assess the differences in the mRNA quantity. Total RNA extraction and reverse transcription were performed using an RNeasy Micro kit (Qiagen, Valencia, CA, USA), according to the manufacturer's instructions and oligo-dT was used as a primer. Real-time PCR analysis was conducted using Fast start Universal SYBR Green Master (Roche Diagnostics Co., Indiannapolis, IN, USA), with an ABI Prism 7500 System (Applied Biosystems, Foster City, CA, USA). We processed 15-30 embryos at a time and GAPDH was used as an endogenous control. The $2^{-\Delta \Delta C t}$ 
method was used to analyze relative changes in gene expression between the groups.

Statistical analysis. All experiments were repeated three times. Prior to significance analyses, all percentage data were subjected to Arcsine transformation. Data were analyzed with paired Student's t-tests and the $\chi^{2}$ test, using SPSS software (SPSS Inc., Chicago, IL, USA). Data are expressed as the mean \pm standard error of the mean. $\mathrm{P}<0.05$ and $\mathrm{P}<0.01$ were considered to indicate a statistically significant difference.

\section{Results}

Expression pattern of Stk40 mRNA in early embryonic stages. Real-time PCR was used to detect Stk40 at different embryonic stages and compare its transcript levels between the stages. cDNA was synthesized from mRNA isolated at the zygote, 2-cell, 4-cell, 8-cell, morula and developing blastocyst stages. For sample analysis, the comparative $2^{-\Delta \Delta \mathrm{CT}}$ method was used to quantify the real-time results for the Stk40 transcripts. All study groups were normalized with GAPDH references and the zygote stage served as the calibrator. Therefore, the efficiencies of target and reference gene detections were similar and the $2^{-\Delta \Delta \mathrm{CT}}$ calculation method was used for relative quantification. Stk40 was expressed at the highest level in the four-cell stage, while the relative level of Stk40 mRNA in the morula or blastocyst stage was only one-tenth that observed at the zygote stage (Fig. 1).

Subcellular localization of Stk40 protein in early embryos. The specificity of antibody to the Stk40 protein was investigated by western blot analysis. The molecular weight of the anti-Stk40 antibody appeared between 43 and $55 \mathrm{kDa}(\sim 50 \mathrm{kDa})$ with mouse ovary samples (Fig. 2A). The subcellular localization of Stk40 protein in early mouse embryos was investigated. The fluorescent staining of Stk40 was observed equally in the cytoplasm and nuclei in all stages of the pre-implantation mouse embryos. However, the most prominent staining appeared in the four-cell stage (Fig. 2B).

Stk40 endoribonuclease-prepared siRNA microinjection and in vitro culture. The inhibitory effect of specific Stk40-targeting siRNA was confirmed using real-time PCR. The injection of the two different Stk40-specific siRNAs (Stk40-1 and Stk40-2) significantly reduced the level of Stk40 mRNA in mouse blastocysts $(\mathrm{P}<0.05$; Fig. 3A). The development of one-cell zygotes in the Stk40 siRNA-injected groups was observably stunted compared with the non-silencing siRNA-injected groups at different time points including 2.5 days (4-cell stage), 3.5 days (morula stage) and 4.5 days (blastocyst stage). In particular, the blastocyst formation rates of the two Stk40-silenced groups showed a significant decrease compared with that of the non-silencing-siRNA injected control groups (Stk40-1 versus control: $65.2 \%$ and $77.0 \%, \mathrm{P}<0.05$; Stk40-2 versus control: $49.8 \%$ and $70.1 \%$, respectively; $\mathrm{P}<0.05$ ) (Fig. 3B).

Increase in Rcn2 expression in Stk40 silenced embryos. The levels of the pluripotency markers Oct4 and Nanog, the extraembryonic endoderm marker Rcn2 and the trophectoderm (TE)-specific gene Cdx2 were assessed using real-time PCR
Table I. Primer sequences.

\begin{tabular}{ll}
\hline Gene name & \multicolumn{1}{c}{ Primer sequence } \\
\hline Stk40 & F: 5'-AGGAACTGGCATTGCTGGAAATAA-3' \\
& R: 5'-TGCTCGGTACCGGTGAGTTG-3' \\
GAPDH & F: 5'-AGGTTGTCTCCTGCGACTTCA-3' \\
& R: 5'-GGGTGGTCCAGGGTTTCTTACT-3' \\
Oct4 & F: 5'-ATGGCATACTGTGGACCTCA-3' \\
& R: 5'-AGCAGCTTGGCAAACTGTTC-3' \\
Nanog & F: 5'-CTCATCAATGCCTGCAGTTTTTCA-3' \\
& R: 5'-CTCCTCAGGGCCCTTGTCAGC-' \\
Rcn2 & F: 5'-GGTCGTTCAGGCAGCTTCATC-3' \\
& R: 5'-CTGGGTCTTCATTTGCAGTTGG-3' \\
Cdx2 & F: 5'-CAGCAGTCCCTAGGAAGCCAA-3' \\
& R: 5'-GTGTGGCAGCCAGCTCACTT-3'
\end{tabular}

Stk40, Serine/threonine protein kinase 40; F, forward; R, reverse; Oct4, octamer-binding transcription factor 4 ; Ren2, reticulocalbin-2.

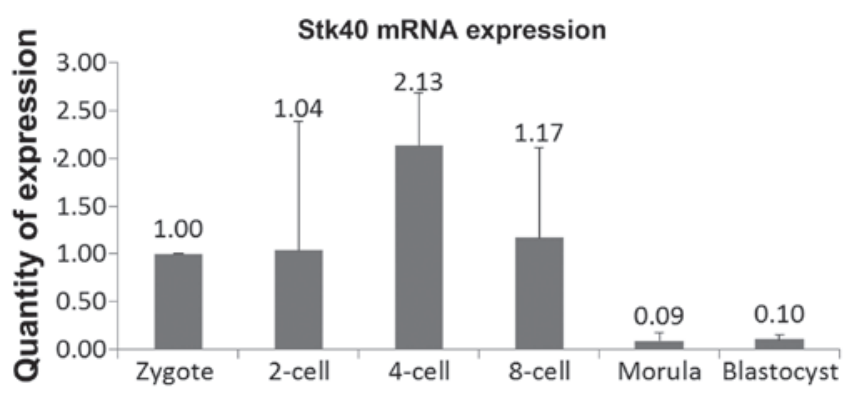

Figure 1. Quantitative polymerase chain reaction analysis of serine/threonine protein kinase 40 (Stk40) mRNA expression levels in all stages of early embryonic development. The $\mathrm{x}$-axis shows the developmental stages, and the $\mathrm{y}$-axis shows the relative expression quantity (mean \pm standard deviation). The Stk40 expression in the one-cell stage served as the calibrator. $\mathrm{x}$-axis: Zygote, $21 \mathrm{~h}$ after human chorionic gonadotropin (HCG) injection; 2-cell stage, $\sim 45 \mathrm{~h}$ after HCG injection; 4-cell stage, $\sim 58 \mathrm{~h}$ after HCG injection; 8-cell stage, $66 \mathrm{~h}$ after HCG injection; morula, $78 \mathrm{~h}$ after HCG injection; and blastocyst, $\sim 96 \mathrm{~h}$ after HCG injection.

in Stk40-silenced embryos and compared with those in the non-silencing siRNA-injected embryos (negative controls). Stk40 siRNA-injected embryos showed significantly increased Rcn 2 and decreased Cdx2 levels (Fig. 4). In every embryonic development stage of each group, at least 35 embryos were observed.

\section{Discussion}

In the present study, Stk40 levels were analyzed in early mouse embryos at different embryonic pre-implantation stages using real-time PCR. The results showed that Stk40 was expressed in all investigated stages; however, the expression level began to decrease markedly from the four-cell stage onwards. Previous studies in mice have demonstrated that the greatest alteration in transcription factor activities occurred between the oneand two-cell stages, at which time zygotic genome activation (ZGA) occurs. Moreover, basic transcription factors, which are 
A

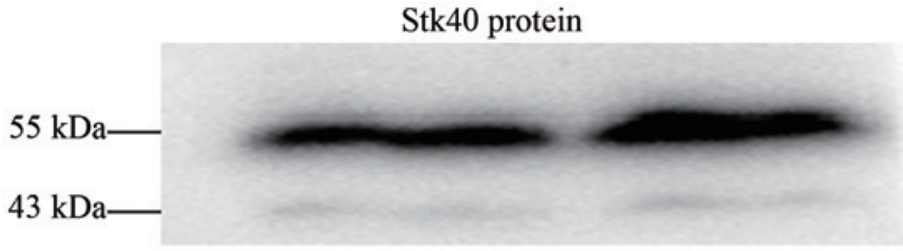

B
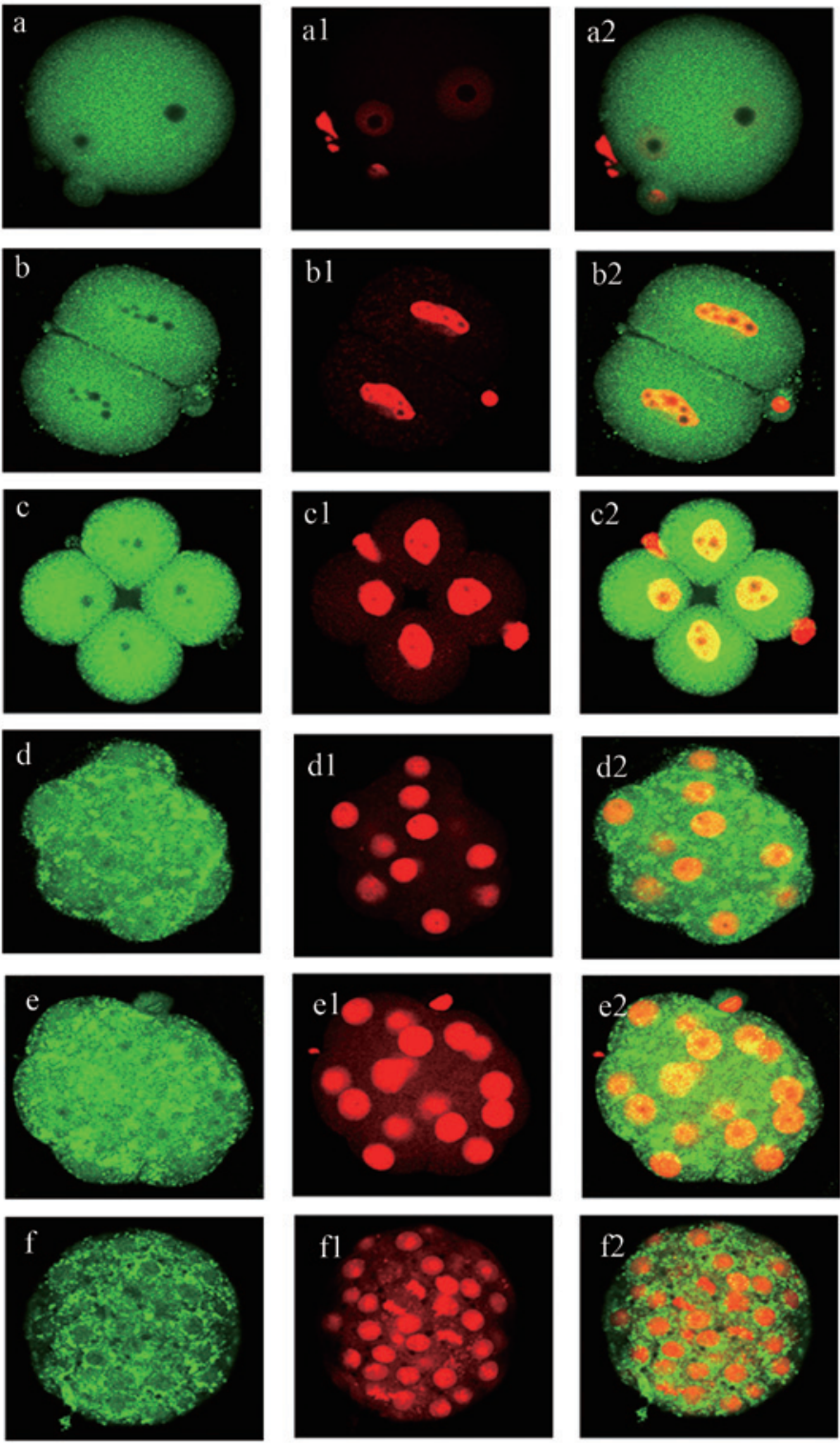

Figure 2. Expression and localization of serine/threonine protein kinase 40 (Stk40) in early mouse embryos. (A) Verification of Stk40 antibody specificity. The antibody identified a band of specific molecular weight as predicted of $\sim 50 \mathrm{kDa}$. The experiment was repeated three times and all showed similar results. (B) Immunofluorescence imaging of Skt40 localization in the (a) zygote (b) 2-cell (c) 4-cell (d) 8-cell (e) morula and (f) blastocyst stage of development. (a1-f1) The corresponding DNA images. Intense fluorescent staining is observed in the nuclei of the embryos in all stages. (a2-f2) Overlapping images of A-F and a1-f1, respectively. Original magnification, x400.

the general components of transcription, increased transiently at the two-cell stage (7). These findings are consistent with data from the present study regarding the expression pattern of Stk40. Thus, the expression peak at the two-cell stage and the subsequent decline suggested that Stk40 may be important in the maternal-to-embryonic transition process. In addition, the subcellular localization of the Stk40 protein confirmed the results of the real-time PCR analysis and showed that there was a translation delay with the most prominent protein staining appearing in the four-cell stage. Furthermore, it was demonstrated that Stk40 downregulation by siRNA markedly decreased the rate of blastocyst formation.

It was observed that the Stk40 transcription factor was expressed in dynamic patterns following fertilization of the oocyte and may be important in embryonic pre-implantation development. Identification of critical regulatory genes is a crucial step in understanding early embryogenesis; thus, the mRNA levels of possible related genes were measured via 


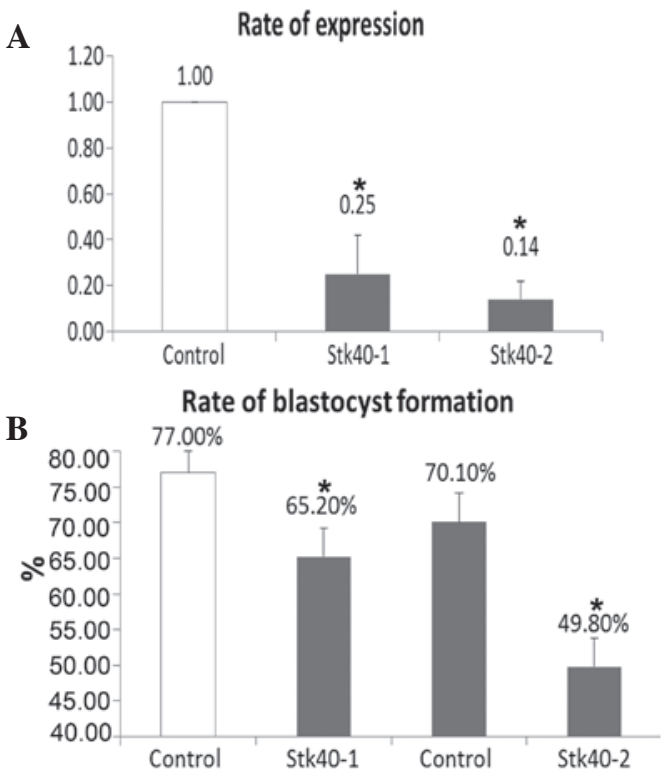

Figure 3. Developmental retardation caused by serine/threonine protein kinase 40 (Stk40) silencing in mouse embryos. (A) Stk40 depletion by Stk40-specific small interfering RNA (siRNA). The efficiency of two Stk40-specific siRNAs on Stk40 mRNA are shown as the ratio of $2^{-\Delta \Delta C T}$ values for Stk40 equalized by GAPDH. (B) Stk40-specific siRNA microinjection retarded early embryonic development. Non-silencing siRNA-injected embryos were used as negative controls. Statistical analysis was performed using data from three repetitions of real-time PCR. Data are presented as the mean \pm standard deviation $(\mathrm{P}<0.05)$. A significant growth inhibitory effect on blastocyst formation was observed in the two Stk40 siRNA-injected groups when compared with the non-silenced siRNA-injected groups.

\section{Related gene expression}

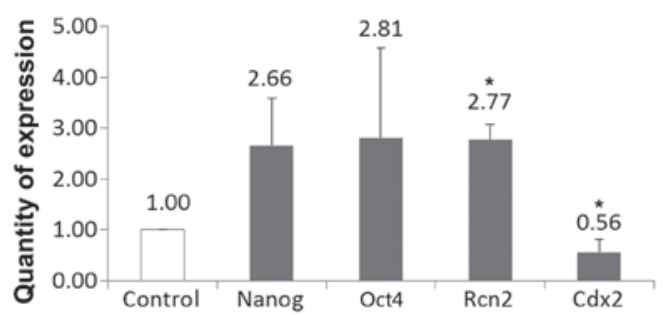

Figure 4. Nanog, octamer-binding transcription factor 4 (Oct4), and reticulocalbin-2 (Ren2) levels increased following serine/threonine protein kinase 40 (Stk40) downregulation, while Cdx2 levels significantly decreased. Non-silencing small interfering RNA (siRNA)-injected embryos were used as controls and the values were set to 1 . At least 35 embryos in every development stage in each group were observed under the same microscope parameters. Data are shown as collective results of all groups. ${ }^{*} \mathrm{P}<0.05$.

real-time, following Stk40-specific siRNA microinjection. This included the pluripotency markers Oct4, Nanog, Rcn2 and $\mathrm{Cdx} 2$. The results showed that Oct4, Nanog and Rcn2 were upregulated and $\mathrm{Cdx} 2$ was downregulated following Stk40-specific siRNA microinjection.

Cell proliferation is an important event in early embryonic development and is largely affected by the regulation of diversified signaling pathways, such as the classic ERK/MAPK signaling pathway (2). This pathway is a critical regulator of cellular processes in adult and developing tissues (17), which exerts its biological effects predominantly through the highly selective activation of ERK1/2 (18). It has been suggested that Stk40 activated Erk1/2 through Ren2 and participated in the ERK/MAPK pathway through Oct4 to control the ExEn lineage differentiation (16). According to the results of the present study, it was suggested that the interaction between Stk40 and the ERK/MAPK signaling pathway influenced the embryonic mouse pre-implantation development. Oct4 and Nanog are known to be pluripotency markers and exhibit restricted expression in pluripotent cell types $(19,20)$. Previous studies indicated that Oct4 was present in all embryonic cells until the late blastocyst stage, gradually disappearing from the TE thereafter (21). Oct4 is highly expressed at the 1- and 2-cell stages in embryos, facilitating ZGA and enhancing maternal mRNA degradation (22). In the present study, the expression pattern of Stk40 was similar to that of Oct4 and, following Stk40 downregulation, increasing Oct4 expression levels appeared in a compensation pattern. Conversely, Nanog expression is initiated in the late stages of pre-implantation embryos and its mRNA is first detected at approximately the eight-cell stage (21). Thus, its expression pattern is opposite to that of Oct4 and Stk40. Notably, Nanog is also an activator of Oct4 through binding to the distal enhancer of Oct4 (19). The results indicated that Nanog expression was upregulated following Stk40 downregulation. This is consistent with a recent study, which demonstrated that Stk40 expression was gradually upregulated when Oct4 expression was rapidly silenced, as Nanog expression was downregulated during this process (16). However, the reciprocal interactions between Stk40 and Nanog remain to be fully evaluated.

It was demonstrated that Stk40 was incapable of promoting the extraembryonic endoderm differentiation when Ren2 was knocked out (16). Rcn2 is a $\mathrm{Ca}^{2+}$-binding protein, known as an extraembryonic endoderm differentiation marker together with Gata6 and Dab2 (23). It was shown that the Stk40 downregulation increased the Rcn 2 expression level $\sim 3$-fold compared with the control group, suggesting that Rcn 2 may function downstream of Stk40; however, the correlation between Ren 2 and embryonic development arrest requires further investigation. $\mathrm{Cdx} 2$ is a known tumor repressor in intestinal epithelium (24). It was previously demonstrated that $\mathrm{Cdx} 2$ is involved in trophectoderm formation at the blastocyst stage in mice and the expression pattern of $\mathrm{Cdx} 2$ during pre-implantation stages is upregulated in 8-16-cell stage embryos (25). Moreover, inactivation of Cdx2 leads to embryonic pre-implantation lethality (26). Therefore $\mathrm{Cdx} 2$ was selected as a blastocyst formation marker for analyzing the interaction with Stk40 at the blastocyst stage. In the present study it was observed that $\mathrm{Cdx} 2$ expression was significantly decreased following Stk40 gene silencing, which significantly affected blastocyst formation. A previous study suggested that $\mathrm{Cdx} 2$ is essential for segregation of the inner cell mass and TE lineages at the blastocyst stage by ensuring repression of Oct4 and Nanog in the TE (27). Thus, it was speculated that low Cdx 2 expression following Stk40 downregulation may have been the reason for Oct 4 and Nanog upregulation. Although the correlation between $\mathrm{Cdx} 2$ and Stk40 was not directly demonstrated, it was suggested that once expression of these genes reached their threshold levels and an imbalance between them occurred, aberrant embryonic development is triggered.

In conclusion, it was suggested that the Stk40 transcription factor may be crucial in mouse embryonic pre-implantation development. Further study of transcription factors may 
provide a reliable molecular biological basis and molecular targeting candidates for improving the efficiency of in vitro embryo culture and enhancing the clinical outcome.

\section{Acknowledgements}

This study was financially supported by the National Natural Science Foundation of China (grant nos. 81100420 and 81270701), the Foundation of Nanjing Medical University (grant no. 2011NJMU210), the Natural Science Foundation of Jiangsu Province (grant no. BK2012520) and the Nanjing Medical Science and Technique Development Foundation (2010NJMU030).

\section{References}

1. Hamatani T, Carter MG, Sharov AA and Ko MS: Dynamics of global gene expression changes during mouse preimplantation development. Dev Cell 6: 117-131, 2004.

2. Miyanari Y and Torres-Padilla ME: Epigenetic regulation of reprogramming factors towards pluripotency in mouse preimplantation development. Curr Opin Endocrinol Diabetes Obes 17: 500-506, 2010.

3. Pantazis P and Bollenbach T: Transcription factor kinetics and the emerging asymmetry in the early mammalian embryo. Cell Cycle 11: 2055-2058, 2012.

4. Sasaki H: Mechanisms of trophectoderm fate specification in preimplantation mouse development. Dev Growth Differ 52: 263-273, 2010

5. Winger Q, Huang J, Auman HJ, Lewandoski M and Williams T: Analysis of transcription factor AP-2 expression and function during mouse preimplantation development. Biol Reprod 75: 324-333, 2006

6. Carey BW, Markoulaki S, Hanna J, et al: Reprogramming of murine and human somatic cells using a single polycistronic vector. Proc Natl Acad Sci USA 106: 157-162, 2009.

7. Kageyama S, Gunji W, Nakasato M, Murakami Y, Nagata M and Aoki F: Analysis of transcription factor expression during oogenesis and preimplantation development in mice. Zygote 15: $117-128,2007$.

8. Saitou M, Kagiwada S and Kurimoto K: Epigenetic reprogramming in mouse pre-implantation development and primordial germ cells. Development 139: 15-31, 2012.

9. Wu FR, Liu Y, Shang MB, et al: Differences in H3K4 trimethylation in in vivo and in vitro fertilization mouse preimplantation embryos. Genet Mol Res 11: 1099-1108, 2012.

10. Palini S, De Stefani S, Scala V, Dusi L and Bulletti C: Epigenetic regulatory mechanisms during preimplantation embryo development. Ann N Y Acad Sci 1221: 54-60, 2011.
11. Bettegowda A, Lee KB and Smith GW: Cytoplasmic and nuclear determinants of the maternal-to-embryonic transition. Reprod Fertil Dev 20: 45-53, 2008.

12. Memili E and First NL: Zygotic and embryonic gene expression in cow: a review of timing and mechanisms of early gene expression as compared with other species. Zygote 8: 87-96, 2000.

13. Zhang JQ, Li XL, Peng Y, Guo X, Heng BC and Tong GQ: Reduction in exposure of human embryos outside the incubator enhances embryo quality and blastulation rate. Reprod Biomed Online 20: 510-515, 2010.

14. Huang J, Teng L, Liu T, et al: Identification of a novel serine/threonine kinase that inhibits TNF-induced NF-kappaB activation and p53-induced transcription. Biochem Biophys Res Commun 309: 774-778, 2003.

15. Kim J, Chu J, Shen X, Wang J and Orkin SH: An extended transcriptional network for pluripotency of embryonic stem cells. Cell 132: 1049-1061, 2008.

16. Li L, Sun L, Gao F, et al: Stk40 links the pluripotency factor Oct4 to the Erk/MAPK pathway and controls extraembryonic endoderm differentiation. Proc Natl Acad Sci USA 107: 1402-1407, 2010.

17. Shvartsman SY, Coppey M and Berezhkovskii AM: MAPK signaling in equations and embryos. Fly (Austin) 3: 62-67, 2009.

18. Rajkumar R, Konishi K, Richards TJ, et al: Genomewide RNA expression profiling in lung identifies distinct signatures in idiopathic pulmonary arterial hypertension and secondary pulmonary hypertension. Am J Physiol Heart Circ Physiol 298: H1235-H1248, 2010.

19. Mitsui K, Tokuzawa $\mathrm{Y}$, Itoh $\mathrm{H}$, et al: The homeoprotein Nanog is required for maintenance of pluripotency in mouse epiblast and ES cells. Cell 113: 631-642, 2003.

20. Nichols J, Zevnik B, Anastassiadis K, et al: Formation of pluripotent stem cells in the mammalian embryo depends on the POU transcription factor Oct4. Cell 95: 379-391, 1998.

21. Dietrich JE and Hiiragi T: Stochastic patterning in the mouse pre-implantation embryo. Development 134: 4219-4231, 2007.

22. Foygel K, Choi B, Jun S, et al: A novel and critical role for Oct4 as a regulator of the maternal-embryonic transition. PLoS One 3: e4109, 2008

23. Gerbe F, Cox B, Rossant J and Chazaud C: Dynamic expression of Lrp2 pathway members reveals progressive epithelial differentiation of primitive endoderm in mouse blastocyst. Dev Biol 313: 594-602, 2008

24. Chawengsaksophak K, James R, Hammond VE, Köntgen F and Beck F: Homeosis and intestinal tumours in $\mathrm{Cdx} 2$ mutant mice. Nature 386: 84-87, 1997.

25. Wang QT, Piotrowska K, Ciemerych MA, et al: A genome-wide study of gene activity reveals developmental signaling pathways in the preimplantation mouse embryo. Dev Cell 6: 133-144, 2004.

26. Chawengsaksophak K, de Graaff W, Rossant J, Deschamps J and Beck F: Cdx2 is essential for axial elongation in mouse development. Proc Natl Acad Sci USA 101: 7641-7645, 2004.

27. Strumpf D, Mao CA, Yamanaka Y, et al: Cdx2 is required for correct cell fate specification and differentiation of trophectoderm in the mouse blastocyst. Development 132: 2093-2102, 2005. 